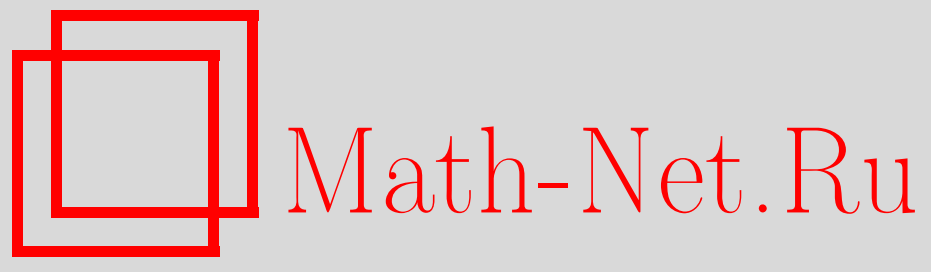

Д. А. Степанов, Заметка о двойственном комплексе разрешения особенностей, УМН, 2006, том 61, выпуск $1,185-186$

DOI: https://doi.org/10.4213/rm1697

Использование Общероссийского математического портала Math-Net.Ru подразумевает, что вы прочитали и согласны с пользовательским соглашением http://www . mathnet.ru/rus/agreement

Параметры загрузки:

IP : 52.6 .47 .48

26 апреля 2023 г., 12:33:58

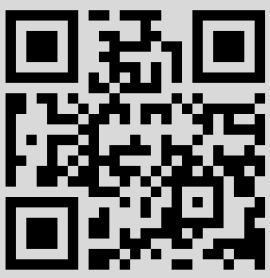




\section{Заметка о двойственном комплексе разрешения особенностей}

\section{Д. А. Степанов}

1. Построение. Двойственный комплекс разрешения особенностей обобщает в высшие размерности понятие графа разрешения двумерной особенности. Пусть $(X, S)$ - росток особенности алгебраического многообразия над полем характеристики $0, \operatorname{dim} X \geqslant 2$. Рассмотрим разрешение $\pi:(Y, E) \rightarrow(X, S)$ особенностей многообразия $X$, исключительное множество $E$ которого - дивизор с простыми нормальными пересечениями. Такое разрешение существует по теореме Хиронаки [1]; следуя терминологии теории минимальных моделей, мы будем называть его лог-разрешением.

Двойственный комплекс $\Gamma(E)$ разрешения $\pi$ - это двойственный клеточный комплекс дивизора $E$ как приводимого многообразия. А именно, пусть $E=\sum_{i=1}^{N} E_{i}-$ разложение дивизора $E$ на простые компоненты $E_{i}$. Тогда $k$-мерные клетки $\Delta_{i_{0} \ldots i_{k}}^{j}$ комплекса $\Gamma(E)$ соответствуют неприводимым компонентам $E_{i_{0} \ldots i_{k}}^{j}$ пересечений $E_{i_{0}} \cap$ $\cdots \cap E_{i_{k}}=\bigcup_{j} E_{i_{0} \ldots i_{k}}^{j}, k=0, \ldots, n-1,1 \leqslant i_{0}<i_{1}<\cdots<i_{k} \leqslant N$, причем каждая клетка $\Delta_{i_{0} \ldots i_{k}}^{j}$ является стандартным $k$-мерным симплексом, вершины которого занумерованы числами $i_{0}, \ldots, i_{k}$. Приклеивающее отображение границы $\partial \Delta_{i_{0} \ldots i_{k}}^{j} k$ мерной клетки к $(k-1)$-мерному остову является симплициальным. Оно отображает грань $\Delta_{i_{0} \ldots \hat{i_{s} \ldots i_{k}}}^{j}$ на тот симплекс $\Delta_{i_{0} \ldots \hat{i_{s} \ldots i_{k}}}^{j^{\prime}}$, для которого $E_{i_{0} \ldots i_{k}}^{j} \cap E_{i_{0} \ldots \hat{i_{s} \ldots i_{k}}}^{j^{\prime}} \neq \varnothing$, и переводит вершину с номером $i_{r}$ грани $E_{i_{0} \ldots \hat{i}_{s} \ldots i_{k}}^{j}$ в вершину с тем же номером $i_{r}$ симплекса $\Delta_{i_{0} \ldots \hat{i_{s}} \ldots i_{k}}^{j^{\prime}}$. Комплекс $\Gamma(E)$ рассматривался в [2].

\section{2. Гомотопический тип двойственного комплекса разрешения как инва- риант особенности.}

Teорема 1. Пусть $\pi^{\prime}:\left(Y^{\prime}, E^{\prime}\right) \rightarrow(X, o)$ u $\pi^{\prime \prime}:\left(Y^{\prime \prime}, E^{\prime \prime}\right) \rightarrow(X, o)$ - два лог-разрешения изолированной особенности $(X, o)$. Тогда топологические пространства $\Gamma\left(E^{\prime}\right)$ и $\Gamma\left(E^{\prime \prime}\right)$ гомотопически эквивалентны.

Этот результат основывается на теореме Абрамовича-Кару-Мацуки-Влодарчика о факторизации бирациональных отображений в логарифмической категории [3].

Так как особенность $(X, o)$ изолирована, можно компактифицировать $X$ до полного многообразия, гладкого вне 0 . Тогда и разрешения $Y^{\prime}$ и $Y^{\prime \prime}$ можно считать полными. По теореме о факторизации бирациональный изоморфизм между $Y^{\prime}$ и $Y^{\prime \prime}$ можно разложить в последовательность

$$
\left(Y^{\prime}, E^{\prime}\right)=\left(Y_{1}, E^{(1)}\right) \stackrel{\psi_{1}}{\rightarrow}\left(Y_{2}, E^{(2)}\right) \stackrel{\psi_{2}}{\rightarrow} \cdots \stackrel{\psi_{k-1}}{\rightarrow--}\left(Y_{k}, E^{(k)}\right)=\left(Y^{\prime \prime}, E^{\prime \prime}\right),
$$

где $E^{(i)}$ - дивизор с простыми нормальными пересечениями в неособом многообразии $Y_{i}, Y_{i} \backslash E^{(i)} \simeq Y_{i+1} \backslash E^{(i+1)}$, а бирациональное отображение $\psi_{i}\left(\right.$ или $\psi_{i}^{-1}$ ) - раздутие гладкого неприводимого центра $C_{i} \subset E^{(i)}$ (или $C_{i+1} \subset E^{(i+1)}$ ), который имеет нормальные пересечения с $E^{(i)}$ (или с $E^{(i+1)}$ ) (так называемого допустимого центра).

Теперь теорема 1 следует из такой леммы.

Лемма. Пусть $\sigma:\left(X^{\prime}, E^{\prime}\right) \rightarrow(X, E)$ - раздутие допустимого центра $C \subset E$ в неособом многообразии $X, E$ - дивизор с простыми нормальными пересечениями. Тогда топологические пространства $\Gamma\left(E^{\prime}\right)$ и $\Gamma(E)$ гомотопически эквивалентны.

ДокАзАтельство. Пусть $E=\sum_{i=1}^{N} E_{i}$ - разложение $E$ на простые дивизоры, а раздуваемый центр $C \subset E_{i}$ при $1 \leqslant i \leqslant l$ и $C \nsubseteq E_{i}$ при $l<i \leqslant N$. Допустим, что $C$ также имеет непустые пересечения с $E_{l+1}, \ldots, E_{r}, l<r \leqslant N$. Возможны два случая.

Работа выполнена при поддержке РФФИ (грант № 05-01-00353) и CRDF (грант № 14841). 
1) $\operatorname{dim} C=n-l(n=\operatorname{dim} X)$, т.е. $C$ совпадает с одной из неприводимых компонент пересечения $E_{1} \cap \cdots \cap E_{l}: C=E_{1 \ldots l}^{1}$. Тогда после раздутия собственные прообразы $E_{1}^{\prime}, \ldots, E_{l}^{\prime}$ дивизоров $E_{1}, \ldots, E_{l}$ имеют пересечение, число неприводимых компонент которого на единицу меньше, чем у пересечения $E_{1} \cap \cdots \cap E_{l}$, но все эти собственные прообразы пересекаются с исключительным дивизором $F$ раздутия $\sigma$. Кроме этого, $F$ пересекается с собственными прообразами $E_{l+1}^{\prime}, \ldots, E_{r}^{\prime}$ дивизоров $E_{l+1}, \ldots, E_{r}$. Поэтому ясно, что комплекс $\Gamma\left(E^{\prime}\right)$ получается из $\Gamma(E)$ барицентрическим разбиением симплекса $\Delta_{1 \ldots l}^{1}$ с центром в точке, соответствующей дивизору $F$, и комплексы $\Gamma\left(E^{\prime}\right)$ и $\Gamma(E)$ оказываются даже гомеоморфными.

$2) \operatorname{dim} C<n-l$, пусть $C \subset E_{1 \ldots l}^{1}$. В этом случае дивизоры $E_{i_{1}}, \ldots, E_{i_{s}}$ имеют непустое пересечение тогда и только тогда, когда и их собственные прообразы $E_{i_{1}}^{\prime}, \ldots, E_{i_{s}}^{\prime}$ имеют непустое пересечение. Поэтому комплекс $\Gamma\left(E^{\prime}\right)$ получается из комплекса $\Gamma(E)$ так. Нужно добавить к $\Gamma(E)$ новую вершину, соответствующую исключительному дивизору $F$ раздутия $\sigma$, и надстроить конусы с вершиной $F$ над всеми максимальными клетками $\Delta_{i_{1} \ldots i_{s}}^{j}$ комплекса $\Gamma(E)$, обладающими свойством $E_{i_{1} \ldots i_{s}}^{j} \cap C \neq \varnothing$. При этом симплекс $\Delta_{F, 1 \ldots l}$, соответствующий пересечению $F \cap E_{1 \ldots l}^{1}$, рассматривается как общий для всех надстроенных конусов. Теперь гомотопическую эквивалентность между $\Gamma\left(E^{\prime}\right)$ и $\Gamma(E)$ можно задать как стягивание надстроенных конусов: переведем вершину $F$ комплекса $\Gamma\left(E^{\prime}\right)$ в любую из вершин $E_{1}, \ldots, E_{l}$ клетки $\Delta_{1 \ldots l}^{1}$ комплекса $\Gamma(E)$, тогда индуцированное симплициальное отображение и будет требуемой гомотопической эквивалентностью.

\section{3. Случай торических особенностей.}

Теорема 2. Пусть о $\in X-$ изолированная особенность торического многообразия $X$. Тогда двойственный комплекс $\Gamma(E)$ любого лог-разрешения $\pi:(Y, E) \rightarrow(X, o)$ гомотопически тривиален (гомотопически эквивалентен точке).

ДокАзАтельство. Можно считать многообразие $X$ аффинным. Тогда его можно представить как торическое многообразие $X=U_{\sigma}=$ Spec $\mathbb{C}[\sigma]$, соответствующее выпуклому полиэдральному конусу $\sigma \subset \mathbb{R}^{n}$. Согласно теореме 1 , достаточно найти гомотопический тип какого-нибудь одного лог-разрешения особенности $(X, o)$. Существует такой веер $\Sigma$, что носитель $\operatorname{supp} \Sigma=\sigma$ и бирациональный морфизм $\pi: Y=X(\Sigma) \rightarrow X$ - лог-разрешение особенностей многообразия $X$ (см. [4]). Рассмотрим сначала двойственный комплекс дивизора $E^{\prime}=\sum T_{i}+E$, где $T_{i}$ - инвариантные дивизоры, соответствующие ребрам $\tau_{i}$ конуса $\sigma$, а $E$ - исключительный дивизор морфизма $\pi$. Простые исключительные дивизоры $E_{i}, E=\sum E_{i}$, соответствуют одномерным конусам веера $\Sigma$, отличным от $\tau_{i}$; дивизоры $E_{i}$ и $E_{j}$ имеют непустое пересечение тогда и только тогда, когда соответствующие одномерные конусы порождают двумерный конус веера $\Sigma$ и т. д. Пусть $L$ - такая гиперплоскость в пространстве $\mathbb{R}^{n}$, что $L \cap \sigma=K-$ компактный полиэдр. Вееру $\Sigma$ тогда отвечает некоторая триангуляция полиэдра $K$. Соответствующий симплициальный комплекс мы тоже будем обозначать через $K$. Теперь ясно, что двойственный комплекс $\Gamma\left(E^{\prime}\right)$ гомеоморфен комплексу $K$ и, очевидно, гомотопически тривиален.

Двойственный комплекс $\Gamma(E)$ получается из $K$ выбрасыванием симплексов, имеющих один из дивизоров $T_{i}$ своей вершиной. Триангуляцию $\Sigma$ можно выбрать как угодно мелкой, поэтому очевидно, что после такого выбрасывания мы получим комплекс, гомотопически эквивалентный исходному, т.е. гомотопически тривиальный.

\section{Список литературы}

[1] H. Hironaka, Ann. of Math. (2), 79 (1964), 109-326. [2] G. L. Gordon, Trans. Amer. Math. Soc., 261:1 (1980), 93-101. [3] D. Abramovich, K. Karu, K. Matsuki, J. Włodarczyk, J. Amer. Math. Soc., 15:3 (2002), 531-572. [4] В. И. Данилов, УМН, 33:2 (1978), 97-154.

Д. А. Степанов (D. A. Stepanov)

Московский государственный технический университет им. Н.Э. Баумана

E-mail: dstepanov@bmstu.ru
Представлено Э. Б. Винбергом Принято редколлегией 01.12.2005 